\title{
The essential role of DOCK8 in humoral immunity
}

\author{
Katrina L. Randall ${ }^{\mathrm{a}, \mathrm{b}}$, Teresa Lambe ${ }^{\mathrm{c}}$, Chris C. Goodnow ${ }^{\mathrm{a}}$ and Richard J. Cornall ${ }^{\mathrm{c}, *}$ \\ a John Curtin School of Medical Research, The Australian National University, Canberra ACT, Australia \\ ${ }^{\mathrm{b}}$ Department of Immunology, The Canberra Hospital, Garran, ACT, Australia \\ ${ }^{\mathrm{c}}$ Nuffield Department of Clinical Medicine, Oxford University, UK
}

\begin{abstract}
The processes that normally generate and maintain adaptive immunity and immunological memory are poorly understood, and yet of fundamental importance when infectious diseases place such a major economic and social burden on the world's health and agriculture systems. Defects in these mechanisms also underlie the many forms of human primary immunodeficiency. Identifying these mechanisms in a systematic way is therefore important if we are to develop better strategies for treating and preventing infection, inherited disease, transplant rejection and autoimmunity. In this review we describe a genome-wide screen in mice for the genes important for generating these adaptive responses, and describe two independent DOCK8 mutant mice strains identified by this screen. DOCK 8 was found to play an essential role in humoral immune responses and to be important in the proper formation of the B cell immunological synapse.
\end{abstract}

Keywords: DOCK8, germinal center, immunodeficiency, DOCK family, guanine exchange factor

\section{Introduction}

The adaptive immune system of higher vertebrates is characterized by the ability to remember previous encounters with antigen and to respond more quickly with higher affinity antibodies, the second time an antigen is encountered. In part, this is due to long-lived bone marrow resident plasma cells and memory $\mathrm{B}$ cells which are formed during the primary response in a specialized structure within the secondary lymphoid tissue known as the germinal center [3]. The exact mechanisms responsible for the development and persistence of these adaptive responses are not known, and a systematic approach to gene discovery may allow new insights into these processes.

Genome wide screening in patients with particular primary immunodeficiencies has identified the genetic cause in many monogenic forms of immunodeficien-

* Corresponding author: Richard J. Cornall, Nuffield Department of Clinical Medicine, Oxford University, UK. Tel.: +44 1865287790 ; Fax: +44 1865 287787; E-mail: richard.cornall@ccmp.ox.ac.uk. cy, while also discovering the role of many genes important in innate and adaptive immune responses [12]. Identifying mouse models of individual immune deficiencies can also be important to inform us about human disease, and provide insights into normal immune function. Characterization of the $\mathrm{SAP}-/-$ mice has allowed new insights into the extent of cellular abnormalities in X-linked lymphoproliferative disease [35, 45], while investigations of WASp -/- mice has provided insights into B cell abnormalities in patients with Wiskott-Aldrich syndrome [39]. Using mouse models to look at the cellular consequences of mutation can be useful in situations in primary immunodeficiency in which it is difficult to differentiate the cellular consequences of the mutation from the consequences of infection arising due to the mutation, or from the effects of antibiotic or antiviral medications used to treat the infections. Mouse models also provide a genetically homogenous environment to study the cellular consequences of the mutation and differentiate these from consequences that may arise due to genetic polymorphisms within a population. 
In this review we discuss the recent identification of murine DOCK8 mutations through the use of a genomewide screen for mice with abnormal vaccination responses, and the consequences for humoral immunity of DOCK8 mutations. We also discuss the DOCK family of proteins in mice, and highlight particular DOCK proteins thought to play a role in the immune system and as well as discussing the parallels with the function that DOCK proteins are thought to play in the nervous system.

\section{The use of ENU mutagenesis to identify novel causes of immunodeficiency}

Long-lived plasma cells and memory B cells produced during adaptive immune responses form the basis of vaccination and protection from disease [3,59], yet the genes, mechanisms and processes behind the production of these important cell types are not fully understood. The genes important in the production of the adaptive immune responses are also likely to be defective in those human primary immunodeficiencies in which a genetic cause has not yet been found (such as in the majority of cases of common variable immunodeficiency (CVID)).

One way to search for these novel genes and mechanisms is to study animals with heritable defects in the immune response that are generated with the chemical mutagen ethylnitrosurea (ENU). Male C57BL/6 mice injected with ENU develop single nucleotide substitutions at a frequency of $\sim 1$ per million base pairs in their spermatogonial stem cells $[52,60]$, which are transmitted to progeny, thus creating libraries of mutant mice that can be screened for heritable phenotypes. Of those that lead to a detectable phenotypic effect, two thirds interfere with the function of a protein due to an amino acid substitution, while one-third lead to aberrant splicing [28]. We have already shown how the characterization of strains from screens for autoimmune disease and lymphocyte development - such as sanroque, tiny and most recently themis $[27,46,61]$ - can reveal new and unexpected information relevant to human disease.

We have now used the same strategy to screen for ENU mutant strains with immunodeficiency detected by an abnormal antibody response to immunization. The immunization screen was devised to screen for mutations affecting the polarization of the immune response to either TH1 or TH2, to look for deficient responses to $\mathrm{T}-$ independent antigens, and lastly defi- ciencies in germinal center formation and affinity maturation in response to $\mathrm{T}$ dependent antigens [60].

The mice were first immunized with chicken gammaglobulin (CGG) coupled to arsonate hapten (ABA) and heat-killed Bordetella pertussis bacilli. The protein CGG normally elicits an IgG1 response, while the B. pertussis elicits an IgG2c response and these antibodies are detected in plasma 14 days after the initial immunization using an enzyme linked immunoassay (ELISA). Mice that produce a robust antibody reaction to CGG but do not respond to B. pertussis are inferred to have a low Th1 response, while those with normal responses to $B$. pertussis and low response to CGG are inferred to have a low $\mathrm{Th} 2$ response. Those in whom the response to both antigens is compromised, are classed as having deficiency of T-dependent immune responses (or mis-injection). To screen for mice with defects in secondary or memory responses, the mice were boosted with ABA-CGG 6 weeks after the first immunization, and antibodies detected by ELISA in plasma collected six days later. In C57BL/6 mice, antibodies to the arsonate hapten only emerge after mutation in germinal centers changes the specificity of the antibody so that it no longer cross-reacts with DNA [23], and antibodies to this particular compound also indicate the presence of germinal centers in the immune response. At the time of the booster injection, the mice were also immunized with NP-Ficoll to test the T-independent responses, with anti-NP antibodies also detected by ELISA six days later [60].

\section{The discovery of DOCK8 immunodeficient mice}

Two novel mutant mouse strains were identified due to abnormalities in the vaccination screen described above - captain morgan ( $\mathrm{cpm}$ ) and primurus (pri). Both were found to have recessive mutations in a poorly characterized $190 \mathrm{kDa}$ guanine nucleotide exchange factor (GEF) called DOCK8.

The cpm mutation was in the exon 20 splice donor sequence and yielded frame shift mutations that eliminated the catalytic GEF domain due to truncating mutations. The GEF domain is also known as DOCK homology region 2 (DHR2) and is one of two conserved regions within all DOCK proteins (the other being DHR1). In pri, an exon 43 mutation caused substitution of a conserved Ser 1827 to Pro, which would be expected to break the predicted alpha helical structure of the same GEF domain. In DOCK9, where the GEF domain structure has recently been solved in complex 


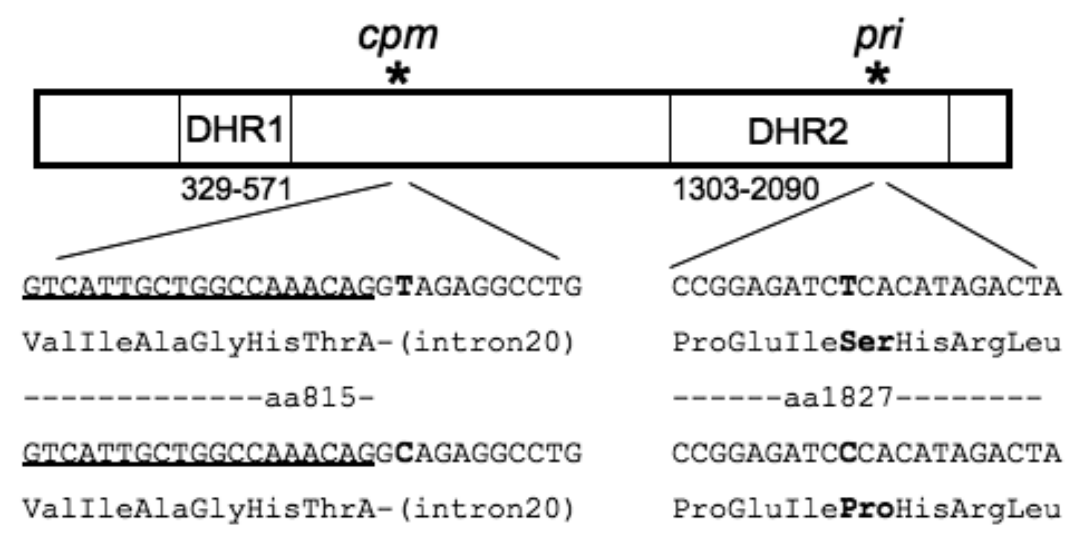

Fig. 1. Two independent mutations in mouse DOCK8. Schematic representation of the structure of murine DOCK8 showing the position of the two conserved protein domains - DHR1 and DHR2. The position of two independent mutations (cpm and pri) that arose through ENU mutagenesis are shown. Underlined text represents the sequence of Exon 20, while bold type indicates the mutated nucleotide. Figure modified from Fig. 3a original published in Nature Immunology; 10: 1283-1291 [53].

with Cdc42 [66], the serine is conserved and lies within alpha helix 6 that forms multiple contacts with Cdc42. The position of the two mutations and the conserved DHR1 and DHR2 domains within the DOCK8 protein are shown in Fig. 1.

The vaccination responses in both cpm and pri mouse strains were further characterized with the antigens used in the initial vaccination screen. Both strains failed to develop a sustained IgG response after a primary immunization with CGG despite having a normal initial immune response and normal polarization to TH1 and TH2 [53]. Both mutations did not seem to compromise the $\mathrm{B}$ cell activation, selection, switching and plasma cell differentiation that occurs in extra-follicular reactions with Th1 and Th2 helper T cells and produces the initial wave of antibody [36]. Both strains of mice, however, failed to make antibodies to arsonate hapten indicating a possible failure of germinal center formation. This was confirmed by the failure of germinal center formation with sheep red blood cell (SRBC) immunization. Homozygous mutations had only small or non-significant effect on T-cell independent extrafollicular B cell responses to NP-Ficoll and compound heterozygote $\mathrm{cpm}+/-\mathrm{pri}+/-$ mice failed to show complementation for the immunization defect, confirming that these phenotypes were due to the DOCK8 mutations [53].

The mutant strains showed no gross abnormalities in the differentiation or numbers of B cells, except for the absence of splenic marginal zone (MZ) B cells, and a reduction in $\mathrm{B} 1 \mathrm{~B}$ cells in the peritoneum, both of which were shown to be B cell intrinsic defects in mixed bone marrow chimeras. In the $\mathrm{T}$ cell compart- ment there was a two-fold reduction in naïve CD8 and CD4 T cells in the blood, spleen and Peyer's patches of both strains, and preservation of cells with an activated/memory CD44hi phenotype [[53], unpublished data.]

Chemotaxis assays carried out using the three main chemotactic factors for germinal center, MZ, and B1 cells - S1P, CXCL12 and CXCL13 - found that mutant and wild-type spleen B cells had similar chemotactic responses. Likewise, in vivo accumulation of $\mathrm{B}$ cells in lymph nodes was indistinguishable between mutant and wildtype (WT) B cells in competitive mixed chimeras. The positioning of B cells within the germinal center depends upon CXCL12 and CXCR4 [2], and positioning of $\mathrm{cpm} / \mathrm{cpm}$ MD4 (conventional IgM/IgD anti-HEL

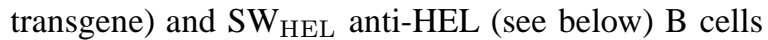
in the germinal center was not different from WT cells B cells in splenic cryosections [[53], unpublished data], confirming that these cells had no major defects in chemotaxis. Mutant B cells also showed no difference compared to wild-type cells in major assays of BCR signaling with normal intracellular calcium flux after stimulation by soluble antigen, and no differences in ERK phosphorylation, induction of the activation markers CD25, CD86 and CD69 on the B cell surface, or induction of DNA synthesis by varying concentrations of soluble HEL or anti-IgM [[53], unpublished data].

\section{DOCK8 is required for germinal center $B$ cell survival}

As described in the previous section, DOCK8 mutant mice were found to have a failure of germinal center 
formation in response to immunization with sheep red blood cells, which normally induce a robust immune response [57], and this failure was found to be B cell intrinsic in bone marrow chimeras [53].

To investigate whether the cell intrinsic abnormality in germinal center B cells in the DOCK8 mutant mice was due to a failure to produce or to sustain the germinal center response, antigen specific B cells were followed through a normal immune response using the $\mathrm{SW}_{\mathrm{HEL}}$ adoptive transfer experimental model. SW $\mathrm{SW}_{\mathrm{HEL}}$ mice carry two transgenes. The first is a rearranged VDJ exon of the HyHEL 10 hybridoma "knocked-in" to the Jh region of the endogenous Ig heavy chain, and the second transgene is a rearranged HyHEL10 kappa light chain at an independent locus [50]. In mice that carry both of the transgenes, between $10-20 \%$ of the B cells express the antigen specific BCR to hen egg lysozyme (HEL). The rest of the B cells express the non-targeted $\mathrm{H}$ chain and hence a polyclonal repertoire of $\mathrm{B}$ cell receptors [9].

Wild-type C57BL/6 male mice (CD45.2) received an intravenous adoptive transfer of $\mathrm{SW}_{\mathrm{HEL}}$ splenocytes containing $10^{5}$ HEL-binding B cells (either pri/pri or wild-type). Included in this injection was $2 \times 10^{8}$ SRBC covalently coupled to $\mathrm{HEL}^{2 X}$ - which binds to the HEL receptor with intermediate affinity [49]. A number of control mice received $10^{5}$ wild-type HELbinding B cells and $2 \times 10^{8}$ SRBC prepared as per the coupled cells but without the addition of HEL (and so the specific antigen for the transgenic B cells was not present). These control mice were designated "mock".

Two and a half days after this transfer, the number of $\mathrm{SW}_{\mathrm{HEL}} \mathrm{B}$ cells was determined by flow cytometry. Equal numbers of cells were found in primurus, wildtype and mock experimental groups. Analysis at day 3.5 and 4.5 showed expansion of the wild-type and primurus $\mathrm{SW}_{\mathrm{HEL}} \mathrm{B}$ cell populations, but this expansion did not occur in the "mock" experimental group.

An important marker of adequate $\mathrm{T}$ cell activation signals to B cells is class switching to $\mathrm{IgG}$ - which occurs in both the extrafollicular plasma cell response and the germinal center response. The same proportion of donor $\mathrm{SW}_{\text {HEL }}$ wild-type and primurus cells were noted to switch to IgG1 in the early phase of the response (day 3.5 and 4.5). This equal proportion of cells switched to IgG1 indicated that there were adequate T cell activation signals to the primurus $\mathrm{SW}_{\mathrm{HEL}} \mathrm{B}$ cells. Chan et al. [11] have shown that switching to IgG1 does not occur if $\mathrm{SW}_{\mathrm{HEL}} \mathrm{B}$ cells are CD40 deficient, and it is also known that the process of switching is linked to the number of divisions that the cells have undergone [25].
In mice that received $\mathrm{SW}_{\mathrm{HEL}} \mathrm{B}$ cells and $\mathrm{SRBC}$, but where the SRBC had not been coated with $\mathrm{HEL}^{2 X}$, no class switching of the antigen specific cells occurred. Although the absolute number of plasma cells produced by pri/pri donor B cells was reduced - at a similar magnitude to the total number of $\mathrm{SW}_{\mathrm{HEL}} \mathrm{B}$ cells - the relative proportion of donor cells that had become plasma cells was comparable between mice that had received wild-type and pri/pri donor cells.

Adoptively transferred primurus $\mathrm{SW}_{\mathrm{HEL}} \mathrm{B}$ cells were able to differentiate to become germinal center $\mathrm{B}$ cells, in contrast to the situation in the primurus mouse in which no germinal centers were made. At day 5, as shown in Fig. 2a, robust germinal center reactions (B220+, GL7+, Fas+ cells) were seen in all mice. However, nine to ten days after adoptive transfer, despite ongoing germinal center reactions in all experimental groups, the antigen specific, donor derived pri/pri $\mathrm{SW}_{\mathrm{HEL}} \mathrm{B}$ cells had almost disappeared dropping to $1 / 20$ of the numbers seen in mice receiving wild-type $\mathrm{SW}_{\mathrm{HEL}} \mathrm{B}$ cells. This almost complete absence was confirmed by immunohistochemistry of splenic cryosections as shown in Fig. 2a. These experiments show that DOCK8 plays an important B cell intrinsic role in the persistence and survival of germinal center B cells.

\section{A defect in the $B$ cell synapse}

Recognition of antigen is known to be important for germinal center B cell survival [3]. In the germinal center, antigen is displayed on the membranes of follicular dendritic cells (FDCs) [44] and when B cells interact with membrane-bound antigen, a B cell immunological synapse is formed [24].

The B cell immunological synapse has been found to consist of a central supramolecular activation complex (cSMAC) where antigen receptors and antigen are clustered on the $\mathrm{B}$ cell and antigen presenting cell respectively, and a peripheral SMAC (pSMAC) where the integrin LFA-1 and its ligand ICAM-1 are concentrated [10]. Integrins are a family of heterodimeric cell adhesion molecules composed of $\alpha$ and $\beta$ subunits important for cell-cell and cell-matrix interactions [6]. Integrins are usually present on the cell surface in an inactive conformation, but can be rapidly activated by conformational changes brought about by external binding ("outside-in signaling") or by activation signals from within the cell brought about by antigen or chemokine receptor stimulation ("inside-out signaling") [1]. The 
A

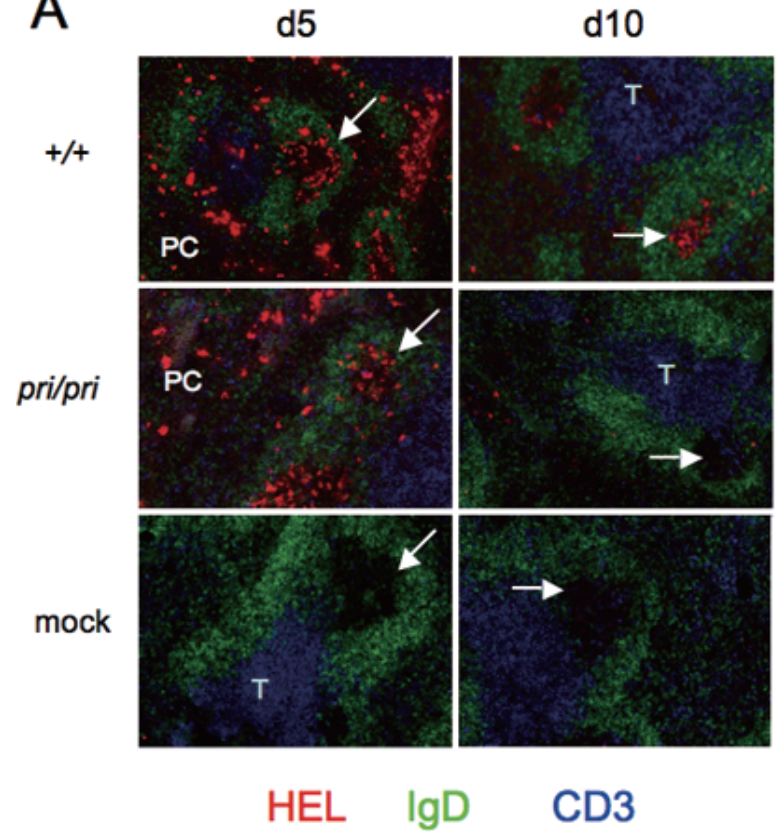

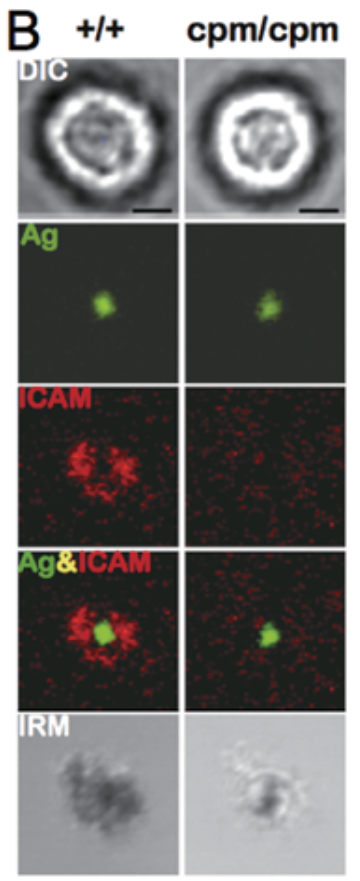
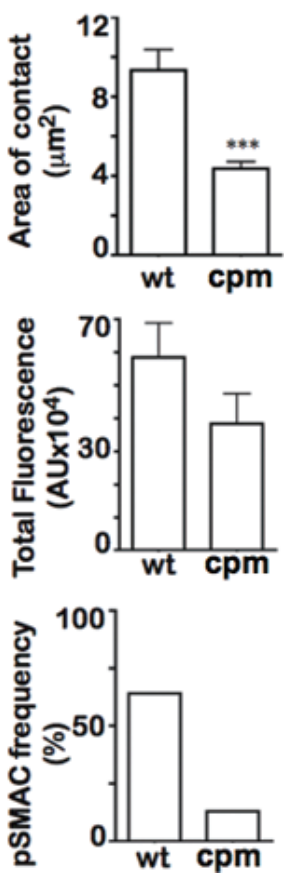

Fig. 2. DOCK8 is essential for germinal center B cell persistence and in the formation of the B cell immunological synapse. (A) Splenic cryosections taken 5 and 10 days after adoptive transfer of $\mathrm{SW}_{\mathrm{HEL}} \mathrm{B}$ cells (either mutant (pri/pri) or wildtype (+/+)) together with HEL ${ }^{2 X}$ conjugated sheep red blood cells (SRBC). One group of mice received SW $\mathrm{HEL}_{\mathrm{B}} \mathrm{B}$ cells and SRBC without HEL (mock). Arrows indicate germinal centers, $\mathrm{T}$, the position of the T cell areas and PC, the extrafollicular plasma cell response. HEL positive B cells are shown in red, IgD positive cells in green and CD3 positive cells in blue. Originally published as Fig. 6a in Randall et al., Nature Immunology; 10: 1283-1291 [53]. (B) MD4 wild-type or DOCK8 mutant naïve B cells were settled into lipid bilayers containing mono-biotinylated HEL as the antigen (green) and Alexa-532 conjugated ICAM-1 (red) and imaged by confocal microscopy after 10 minutes of interaction. Differential interference contrast (DIC), fluorescence and interference reflection microscopy (IRM) images of representative cells are shown. Quantification of the area of B cell contact with the bilayer, the relative amounts of antigen accumulated (expressed as a sum of fluorescence) and the percentage of B cells forming a pSMAC. Columns are means and error bars standard error of the mean. Originally published as Fig. 7a in Randall et al., Nature Immunology; 10: 1283-1291 [53] (generated by Bebhinn Treanor, CRUK).

main integrins on $\mathrm{B}$ cells are LFA-1, which binds to ICAM $-1,-2,-3$ and -5 , and VLA-4, which binds to VCAM-1 and fibronectin. The ligands for LFA-1 and VLA-4 are found on endothelial cells, leukocytes including B cells, dendritic cells and follicular dendritic cells [34] and are upregulated on follicular dendritic cells in the germinal center [5].

The recruitment and activation of the integrin components of the immunological synapse is a consequence of antigen signaling through the BCR, but the exact pathway is still not known. Recent work has shown that the proper formation of the B cell immune synapse after antigen stimulation is dependent on signaling by src family tyrosine kinases, and is disrupted in mice treated with inhibitors of these kinases, and is also disrupted in mice carrying mutations of PI3K, Vav and Rac2 [7]. Disruptions in any of these signaling components affect the BCR dependent activation of Rap1. Rap1 is an important regulator of cell polarity and actin [32].
DOCK8 mutant B cells were assessed for their ability to form a B cell immune synapse using the in vitro assay developed by Facundo Batista and his laboratory [7]. As shown in the left panel of Fig. 2b, addition of wild-type HEL specific B cells led to rearrangement of the antigen and ICAM to reflect the formation of the immunological synapse, with antigen clustered centrally in a cSMAC surrounded by a ring of ICAM. The right hand panel of Fig. $2 b$ shows the parallel analysis of Dock8 deficient ( $\mathrm{cpm} / \mathrm{cpm}$ ) HEL specific cells. Antigen clustering into a cSMAC occurred but there was no surrounding ring of ICAM indicating that the pSMAC had not formed.

Integrins have been found to provide important costimulatory signals for germinal center cell survival in vitro notably via the integrin ligand ICAM1 [30] Therefore, the inability to fully engage with FDCs through the failure to form a proper immune synapse might explain the failure of germinal center B cell survival in the DOCK8 mutants. 


\section{The DOCK gene family}

The identification of the role of DOCK8 in germinal center B cell survival and in the formation of the B cell synapse highlights the importance of the DOCK family of RhoGTPase GEFs in cellular function. RhoGTPases exert their effect only when bound with GTP and rapidly lose their activity as they hydrolyze GTP to GDP. Two principal groups of proteins act as GEFs for Rho GTPases and activate them by exchanging GDP for GTP [15], the Dbl-homology domain containing proteins such as Vav1 (classical GEFs) and DOCK family proteins (non-classical GEFs).

There are 11 mammalian members of the DOCK family divided into four groups based on the degree of homology of two conserved DHR domains [13,38]:

- DOCK-A - containing DOCK180 (also known as DOCK1), DOCK2 and DOCK5

- DOCK-B - containing DOCK3 (also known as MOA) and DOCK4

- DOCK-C - containing DOCK6 (also known as Zir1), DOCK7 (also known as Zir2) and DOCK8 (also known as Zir3)

- DOCK-D - containing DOCK9 (also known as zizimin1), DOCK10 (also known as zizimin3) and DOCK11 (also known as zizimin 2).

A number of these proteins have been found to be critical activators of Rho/Rac/Cdc42 small GTPase proteins in worms, flies and mammals [15,63].

The first family member, DOCK180, was cloned in 1996 during a search for proteins interacting with the proto-oncogene Crk28 [26]. DOCK180's C. elegans orthologue Ced-5 controls cell migration and phagocytosis [64], whilst the Drosophila orthologue, Myoblast city, is essential for myoblast fusion and dorsal closure [18]. DOCK8 was originally isolated in a yeast two-hybrid screen for binding partners of Cdc42 (though this was not confirmed in a pull-down assay) and found to localize at lamellipodia of fibroblasts [55].

As described above, the proteins of the DOCK family are classified according to their two DOCK homology domains. The DHR1 domain of DOCK1 has been shown to bind to the lipid PtdIns(3,4,5)P3 (PIP3) in the cell membrane and it is thought that this binding mediates Rac-dependent actin re-organization of the leading edge of the cell during migration [14]. The DHR1 domains of other DOCK proteins have also been found to bind to PIP3 in in vitro studies - including DOCK2, DOCK7 [15] and DOCK4 and its splice variants [29]. The DHR2 domain binds to Rho-family
GTPase-proteins (Rac, Cdc42) and provides catalytic GEF activity to stimulate these proteins and promote integrin reorganization and adhesion, lamellipodia formation, cell polarization, phagocytosis or cell fusion [15].

Dock 8 mRNA is expressed at ten times greater quantity in B and T lymphocytes than in other tissues (BioGPS, SymAtlas) and our studies of DOCK8 immunodeficient mice, which appear normal in growth and behaviour, indicate that it plays a relatively specialized role within the immune system.

\section{Other murine DOCK family members with roles in the immune system}

The specificity of Dock 8 functions within the immune system contrast with those of Dock2, which is the other DOCK family member characterized in detail with respect to its function in the immune system. DOCK2 mutations in mice result in lymphopenia and disrupted lymphoid architecture with crippled B cell migration to lymph nodes and a general defect in chemotaxis [19].

Despite other differences, Dock 8 and Dock 2 deficient mice share the absence of MZ B cells, and DOCK 2 and other guanine exchange factor proteins have also been shown to play a role in integrin signaling.

The DOCK $2^{-/-}$strain has not been assessed for B cell synapse formation, but DOCK2 has been shown to be important for integrin activation in response to chemokine signaling in B cells [48]. DOCK2 has however been shown to be important for proper $\mathrm{T}$ cell synapse formation after activation by antigen. It is essential for translocation of TCR and lipid rafts into the synapse, but is not essential for proper placement of PKC- $\theta$ and LFA-1 in the synapse [56].

The localization of MZ B cells in the marginal zone depends upon both expression of LFA- 1 and $\alpha 4 \beta 1$ integrins [33], and the high expression of S1P1 and S1P3 receptors, whose activation is thought to counteract CXCL13 chemoattractant signals from the follicles [37]. MZ development is also tightly regulated by interactions between Notch2 and delta-like 1 receptors [58] and by the strength of BCR signaling, which may involve positive and/or negative selection by selfantigen [51]. In this context, DOCK8 might be involved in BCR signaling, or forms of signaling and adhesion critical to MZ B cell development or survival, perhaps in a manner similar to that in germinal center B cells. It is also possible that cytoskeletal changes due to 
DOCK8 mutation affect cell-cell contacts or the morphology of the MZ cells. Relative and absolute defects in MZ B cells are shared by several mice lacking signaling molecules involved in cytoskeletal changes and cell localization including Rac2 [16], and Lsc (orthologue of human p115RhoGEF). Lsc is part of the signaling cascade downstream of $\mathrm{G} \alpha_{12}$ and $\mathrm{G} \alpha_{13}$. (part of the $\mathrm{S} 1 \mathrm{P}$ receptor) and important for lymphocyte chemotaxis [20] and a decrease in marginal zone $\mathrm{B}$ cells is also seen with conditional deletions of $\mathrm{G} \alpha_{12}$ and $\mathrm{G} \alpha_{13}$ in $\mathrm{B}$ cells [54] and Pyk-2 $2^{-/-}$mice [22], another component of this signaling pathway.

Other members of the DOCK family have also been shown to have possible functions in the immune system. DOCK11 (also known as zizimin2) was cloned in a screen for genes enriched in germinal center B cells using a cDNA library derived from C57BL/6 mice. DOCK11 was found to be expressed at 2.6 fold higher levels in germinal center cells as opposed to nongerminal center B cells [47], but its role in these cells has not been further elucidated and the consequences of mutating this gene in mice is not known. DOCK10 was identified as an IL-4 inducible gene in human chronic lymphocytic leukemia (CLL) and has been found to be expressed predominantly in peripheral blood leukocytes [67], but again the consequences of its absence have not been studied.

\section{Parallels between GEF functions in the central nervous system and immune system}

RNA microarray studies of genes expressed by germinal center B cells have found that a large proportion of genes expressed by these cells are also involved in axon growth and guidance [68], and we know that germinal center cells have been shown to take on a dendritic-like appearance after in vitro activation [68] and in two photon studies of the germinal center [4]. It is therefore interesting to note that a number of DOCK family proteins are thought to play an important signaling role within the nervous system, particularly in the formation of axons and dendrites [41].

Does DOCK8 also play a role within the nervous system? Heterozygous deletions in DOCK 8 have been reported in two people with mental retardation [21], although this effect might be explained by defects in the adjacent gene with a well established role in mental retardation, ANKRD15 [31], as no neurological or cognitive impairment has been described in people with homozygous mutations and immunodeficiency $[17,69]$.
The DOCK-C family, to which DOCK8 belongs, also contains DOCK6 and DOCK7, both of which have been found to have possible roles in the nervous system. DOCK6 has been shown in GTPase binding studies to have dual specificity for both Rac1 and Cdc42, and when the DHR2 domain of DOCK6 was transfected into COS cells it led to lamellipodia and filopodia formation. Neurite outgrowth in the N1E-115.5 cell line was shown to be associated with higher expression of Dock6 and addition of siRNA specific for Dock6 was shown to prevent neurite outgrowth in this cell line [40]. The exact role of Dock6 in vivo has not yet been determined.

DOCK7 was isolated in a yeast two-hybrid screen for Rac1 binding partners in brain tissue and this binding has been confirmed by GTPase assays. It does not have dual GTPase specificity and does not bind to Cdc42. DOCK7 has been found in a polarized distribution in hippocampal neurons and has been found to be essential for normal axonal growth - with decreased DOCK7 due to siRNA knock-down causing loss of axons, while over-expression gives rise to multiple axons [62]. DOCK7 has also been shown to be important for control of Schwann cell migration through binding of ErbB2 [65] and has been found to associate with the protein product of the tumor suppressor genes TSC 1 and TSC2. Mutations in the TSC1 and TSC2 genes give rise to the human disease tuberous sclerosis characterized by the formation of multiple benign tumors [43]. However, more recently, two strains of mice with mutations in Dock7 have been described in one strain, misty, the mutation arose spontaneously and in the other a splicing error arose by ENU mutagenesis. Both strains of mice show no overt neurological abnormalities but have a general hypopigmentation and show white spotting of the coat [8]. The differences between the in vivo and in vitro phenotype may be due to the nature of the mutation that has arisen in the two mice strains, which may leave the guanine exchange function intact, or due to redundancy in the function of this protein within the nervous system. Interesting$\mathrm{ly}$, the proteins in the DOCK-C family that have been associated with nerve growth abnormalities and neural development rely on many of the same molecules as those found in lymphocyte signaling pathways such as Rac, Vav2 and TIAM [41].

DOCK3 has also recently been found to play a role in the nervous system affecting axonal length both in vitro and in vivo. Primary hippocampal neurons transfected with a plasmid containing Dock3 showed increased axonal growth, while transgenic mice expressing increased Dock3 had structurally normal neural tissues, but increased axon length [42]. 


\section{Conclusion}

Mutation of the DOCK8 protein in mice has profound effects on humoral immunity with a failure to sustain the antibody response and failure of germinal center B cell persistence. Mice with Dock 8 mutations also have absent marginal zone B cells, and decreased peritoneal B1 cell and naïve T cell numbers. While B cell signaling is not affected in measures such as proliferation, calcium flux and expression of activation markers, Dock 8 deficiency prevents formation of the normal B cell immune synapse, affecting the pSMAC. This is likely to limit B cell survival during the germinal center response when survival is determined by competitive interaction of B cells with small amounts of antigen and other survival factors on FDCs. Further study of DOCK8 mutant mice will help to elucidate the mechanisms underlying this selection point, Other studies of Dock 8 mutant mice, including assessments of T cell proliferation, signaling and cell survival may help to further elucidate the mechanisms of immunodeficiency arising in patients with combined immunodeficiency due to homozygous mutations of DOCK8.

\section{Acknowledgements}

The authors wish to acknowledge their co-authors on previous publications, particularly Drs. Bebhinn Treanor and Facundo Batista for data discussed in this paper.

\section{References}

[1] C.L. Abram and C.A. Lowell, Convergence of immunoreceptor and integrin signaling, Immunol Rev 218 (2007), 29-44.

[2] C.D.C. Allen et al., Germinal center dark and light zone organization is mediated by CXCR4 and CXCR5, Nat Immunol 5 (2004), 943-952.

[3] C.D.C. Allen, T. Okada and J.G. Cyster, Germinal-center organization and cellular dynamics, Immunity 27 (2007), 190202.

[4] C.D.C. Allen, T. Okada, H.L. Tang and J.G. Cyster, Imaging of germinal center selection events during affinity maturation, Science 315 (2007), 528-531.

[5] C.D.C. Allen and J.G. Cyster, Follicular dendritic cell networks of primary follicles and germinal centers: phenotype and function, Semin Immunol 20 (2008), 14-25.

[6] E. Arana, N.E. Harwood and F.D. Batista, Regulation of integrin activation through the B-cell receptor, Journal of Cell Science 121 (2008), 2279-2286.

[7] E. Arana et al., Activation of the small GTPase Rac2 via the B cell receptor regulates B cell adhesion and immunologicalsynapse formation, Immunity $\mathbf{2 8}$ (2008), 88-99.
[8] A.L. Blasius et al., Mice with mutations of Dock7 have generalized hypopigmentation and white-spotting but show normal neurological function, Proceedings of the National Academy of Sciences of the United States of America 106 (2009), 27062711.

[9] R. Brink, T.G. Phan, D. Paus and T.D. Chan, Visualizing the effects of antigen affinity on T-dependent B-cell differentiation, Immunology \& Cell Biology 86 (2008), 31-39.

[10] Y.R. Carrasco, S.J. Fleire, T. Cameron, M.L. Dustin and F.D. Batista, LFA-1/ICAM-1 Interaction Lowers the Threshold of B Cell Activation by Facilitating B Cell Adhesion and Synapse Formation, Immunity 20 (2004), 589-599.

[11] T.D. Chan et al., Antigen Affinity Controls Rapid T-Dependent Antibody Production by Driving the Expansion Rather than the Differentiation or Extrafollicular Migration of Early Plasmablasts, J Immunol 183 (2009), 3139-3149.

[12] M.E. Conley et al., Primary B cell immunodeficiencies: comparisons and contrasts, Annu Rev Immunol 27 (2009), 199227.

[13] J.-F. Cote and K. Vuori, Identification of an evolutionarily conserved superfamily of DOCK180-related proteins with guanine nucleotide exchange activity, J Cell Sci 115 (2002), 4901-4913.

[14] J.-F. Cote, A.B. Motoyama, J.A. Bush and K. Vuori, A novel and evolutionarily conserved PtdIns(3,4,5)P3-binding domain is necessary for DOCK180 signalling.[see comment], Nature Cell Biology 7 (2005), 797-807.

[15] J.-F. Cote and K. Vuori, GEF what? Dock180 and related proteins help Rac to polarize cells in new ways, Trends in Cell Biology 17 (2007), 383-393.

[16] B.A. Croker et al., The Rac2 guanosine triphosphatase regulates B lymphocyte antigen receptor responses and chemotaxis and is required for establishment of B-1a and marginal zone B lymphocytes, J Immunol 168 (2002), 3376-3386.

[17] K.R. Engelhardt et al., Large deletions and point mutations involving the dedicator of cytokinesis 8 (DOCK8) in the autosomal-recessive form of hyper-IgE syndrome, J Allergy Clin Immunol 124 (2009), 1289-1302 e4.

[18] M.R. Erickson, B.J. Galletta and S.M. Abmayr, Drosophila myoblast city encodes a conserved protein that is essential for myoblast fusion, dorsal closure, and cytoskeletal organization, J Cell Biol 138 (1997), 589-603.

[19] Y. Fukui et al., Haematopoietic cell-specific CDM family protein DOCK2 is essential for lymphocyte migration, Nature 412 (2001), 826-831.

[20] I. Girkontaite et al., Lsc is required for marginal zone B cells, regulation of lymphocyte motility and immune responses, Nat Immunol 2 (2001), 855-862.

[21] B.L. Griggs, S. Ladd, R.A. Saul, B.R. DuPont and A.K. Srivastava, Dedicator of cytokinesis 8 is disrupted in two patients with mental retardation and developmental disabilities, Genomics 91 (2008), 195-202.

[22] R. Guinamard, M. Okigaki, J. Schlessinger and J.V. Ravetch, Absence of marginal zone B cells in Pyk-2-deficient mice defines their role in the humoral response.[see comment], Nat Immunol 1 (2000), 31-36.

[23] S. Hande, E. Notidis and T. Manser, Bcl-2 obstructs negative selection of autoreactive, hypermutated antibody $\mathrm{V}$ regions during memory B cell development, Immunity 8 (1998), 189198.

[24] N.E. Harwood and F.D. Batista, Early events in B cell activation, Aпnи Rev Immunol 28 (2010), 185-210.

[25] J. Hasbold, L.M. Corcoran, D.M. Tarlinton, S.G. Tangye and P.D. Hodgkin, Evidence from the generation of immunoglobu- 
lin G-secreting cells that stochastic mechanisms regulate lymphocyte differentiation, Nat Immunol 5 (2004), 55-63.

[26] H. Hasegawa et al., DOCK180, a major CRK-binding protein, alters cell morphology upon translocation to the cell membrane, Mol Cell Biol 16 (1996), 1770-1776.

[27] A.L. Johnson et al., Themis is a member of a new metazoan gene family and is required for the completion of thymocyte positive selection, Nat Immunol 10 (2009), 831-839.

[28] M.J. Justice, J.K. Noveroske, J.S. Weber, B. Zheng and A. Bradley, Mouse ENU mutagenesis, Human Molecular Genetics 8 (1999), 1955-1963.

[29] A. Kanai et al., Identification of DOCK4 and its splicing variant as PIP3 binding proteins, IUBMB Life 60 (2008), 467-472.

[30] G. Koopman et al., Adhesion through the LFA-1 (CD11a/CD18)-ICAM-1 (CD54) and the VLA-4 (CD49d)VCAM-1 (CD106) pathways prevents apoptosis of germinal center B cells, J Immunol 152 (1994), 3760-3767.

[31] I. Lerer et al., Deletion of the ANKRD15 gene at 9p24.3 causes parent-of-origin-dependent inheritance of familial cerebral palsy, Human Molecular Genetics 14 (2005), 3911-3920.

[32] K.B.L. Lin et al., The rap GTPases regulate B cell morphology, immune-synapse formation, and signaling by particulate B cell receptor ligands, Immunity 28 (2008), 75-87.

[33] T.T. Lu and J.G. Cyster, Integrin-Mediated Long-Term B Cell Retention in the Splenic Marginal Zone, Science 297 (2002), 409-412.

[34] B.-H. Luo, C.V. Carman and T.A. Springer, Structural basis of integrin regulation and signaling, Апnи Rev Immunol $\mathbf{2 5}$ (2007), 619-647.

[35] C.S. Ma, K.E. Nichols and S.G. Tangye, Regulation of cellular and humoral immune responses by the SLAM and SAP families of molecules, Annu Rev Immunol 25 (2007), 337-379.

[36] I.C.M. MacLennan et al., Extrafollicular antibody responses, Immunol Rev 194 (2003), 8-18.

[37] M. Matloubian et al., Lymphocyte egress from thymus and peripheral lymphoid organs is dependent on S1P receptor 1, Nature 427 (2004), 355-360.

[38] N. Meller, S. Merlot and C. Guda, CZH proteins: a new family of Rho-GEFs, J Cell Sci 118 (2005), 4937-4946.

[39] A. Meyer-Bahlburg et al., Wiskott-Aldrich syndrome protein deficiency in B cells results in impaired peripheral homeostasis. [see comment], Blood 112 (2008), 4158-4169.

[40] Y. Miyamoto, J. Yamauchi, A. Sanbe and A. Tanoue, Dock6, a Dock-C subfamily guanine nucleotide exchanger, has the dual specificity for Rac1 and $\mathrm{Cdc} 42$ and regulates neurite outgrowth, Experimental Cell Research 313 (2007), 791-804.

[41] Y. Miyamoto and J. Yamauchi, Cellular signaling of Dock family proteins in neural function, Cell Signal 22 (2010), 175182.

[42] K. Namekata et al., Dock3 induces axonal outgrowth by stimulating membrane recruitment of the WAVE complex, Proc Natl Acad Sci U S A 107 (2010), 7586-7591.

[43] M. Nellist, P.C. Burgers, A.M.W. van den Ouweland, D.J.J. Halley and T. M. Luider, Phosphorylation and binding partner analysis of the TSC1-TSC2 complex, Biochemical and Biophysical Research Communications 333 (2005), 818-826.

[44] M.S. Neuberger et al., Memory in the B-cell compartment: antibody affinity maturation, Philosophical Transactions of the Royal Society of London - Series B: Biological Sciences 355 (2000), 357-360.

[45] K.E. Nichols et al., Regulation of NKT cell development by SAP, the protein defective in XLP.[see comment], Nature Medicine 11 (2005), 340-345.

[46] A. Nijnik et al., DNA repair is limiting for haematopoietic stem cells during ageing, Nature 447 (2007), 686-690.

[47] Nishikimi et al., Zizimin2: a novel, DOCK180-related Cdc42 guanine nucleotide exchange factor expressed predominantly in lymphocytes, FEBS Letters 579 (2005), 1039-1046.

[48] C. Nombela-Arrieta et al., Differential Requirements for DOCK2 and Phosphoinositide-3-Kinase [gamma] during T and B Lymphocyte Homing, Immunity 21 (2004), 429-441.

[49] D. Paus et al., Antigen recognition strength regulates the choice between extrafollicular plasma cell and germinal center B cell differentiation, J Exp Med 203 (2006), 1081-1091.

[50] T.G. Phan et al., B Cell Receptor-independent Stimuli Trigger Immunoglobulin (Ig) Class Switch Recombination and Production of IgG Autoantibodies by Anergic Self-Reactive B Cells, J Exp Med 197 (2003), 845-860.

[51] S. Pillai, A. Cariappa and S.T. Moran, Marginal zone B cells, Annu Rev Immunol 23 (2005), 161-196.

[52] M.M. Quwailid et al., A gene-driven ENU-based approach to generating an allelic series in any gene, Mamm Genome $\mathbf{1 5}$ (2004), 585-591.

[53] K.L. Randall et al., Dock8 mutations cripple B cell immunological synapses, germinal centers and long-lived antibody production, Nat Immunol 10 (2009), 1283-1291.

[54] S. Rieken et al., G12/G13 family G proteins regulate marginal zone B cell maturation, migration, and polarization, J Imтипо 177 (2006), 2985-2993.

[55] A. Ruusala and P. Aspenstrom, Isolation and characterisation of DOCK8, a member of the DOCK180-related regulators of cell morphology, FEBS Letters 572 (2004), 159-166.

[56] T. Sanui et al., DOCK2 is essential for antigen-induced translocation of TCR and lipid rafts, but not PKC-theta and LFA-1, in T cells, Immunity 19 (2003), 119-129.

[57] S.M. Shinall, M. Gonzalez-Fernandez, R.J. Noelle and T.J. Waldschmidt, Identification of murine germinal center B cell subsets defined by the expression of surface isotypes and differentiation antigens, J Immunol 164 (2000), 5729-5738.

[58] J.B. Tan et al., Lunatic and manic fringe cooperatively enhance marginal zone B cell precursor competition for deltalike 1 in splenic endothelial niches.[see comment], Immunity 30 (2009), 254-263.

[59] D.M. Tarlinton, Evolution in miniature: selection, survival and distribution of antigen reactive cells in the germinal centre, Immunology \& Cell Biology 86 (2008), 133-138.

[60] C.G. Vinuesa and C.C. Goodnow, Illuminating autoimmune regulators through controlled variation of the mouse genome sequence, Immunity 20 (2004), 669-679.

[61] C.G. Vinuesa et al., A RING-type ubiquitin ligase family member required to repress follicular helper $\mathrm{T}$ cells and autoimmunity, Nature 435 (2005), 452-458.

[62] M. Watabe-Uchida, K.A. John, J.A. Janas, S.E. Newey and L. Van Aelst, The Rac Activator DOCK7 Regulates Neuronal Polarity through Local Phosphorylation of Stathmin/Op18, Neuron 51 (2006), 727-739.

[63] K. Wennerberg and C.J. Der, Rho-family GTPases: it's not only Rac and Rho (and I like it), J Cell Sci 117 (2004), 13011312.

[64] Y.C. Wu and H.R. Horvitz, C. elegans phagocytosis and cellmigration protein CED-5 is similar to human DOCK180, $\mathrm{Na}$ ture 392 (1998), 501-504.

[65] J. Yamauchi, Y. Miyamoto, J.R. Chan and A. Tanoue, ErbB2 directly activates the exchange factor Dock7 to promote Schwann cell migration, J Cell Biol 181 (2008), 351-365.

[66] J. Yang, Z. Zhang, S.M. Roe, C.J. Marshall and D. Barford, Activation of Rho GTPases by DOCK exchange factors is 
mediated by a nucleotide sensor, Science 325 (2009), 13981402.

[67] E. Yelo et al., Dock10, a novel CZH protein selectively induced by interleukin-4 in human B lymphocytes, Molecular Immunology 45 (2008), 3411-3418.
[68] D. Yu et al., Axon growth and guidance genes identify Tdependent germinal centre B cells.[see comment], Immunology \& Cell Biology 86 (2008), 3-14.

[69] Q. Zhang et al., Combined immunodeficiency associated with DOCK8 mutations, N Engl J Med 361 (2009), 2046-2055. 


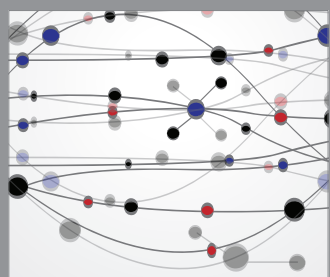

The Scientific World Journal
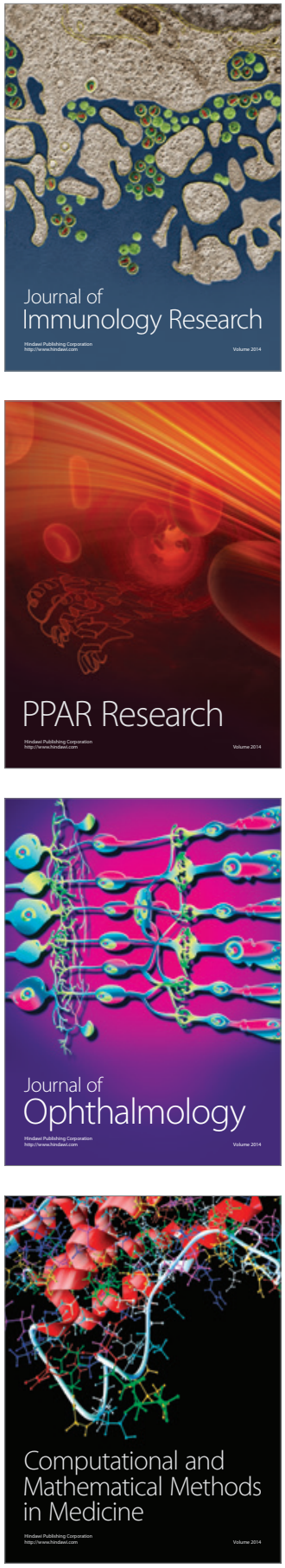

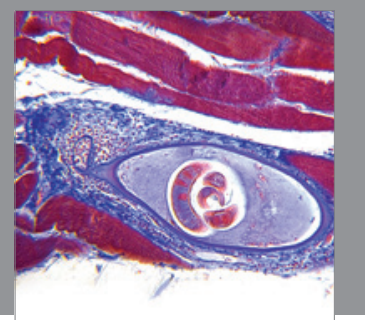

Gastroenterology

Research and Practice
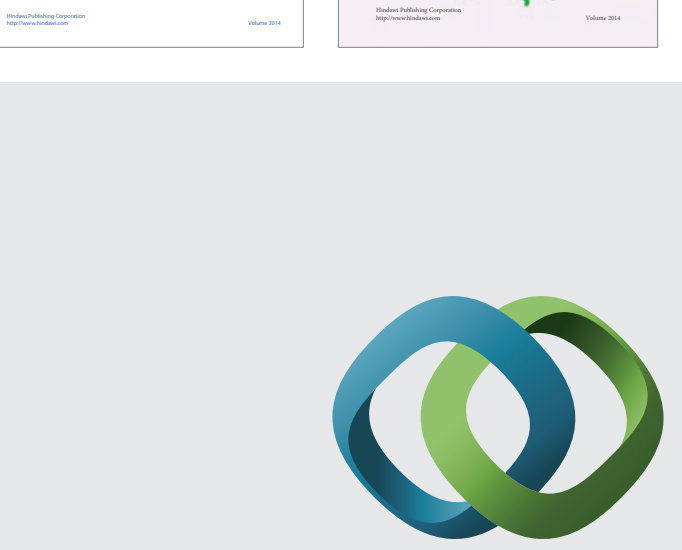

\section{Hindawi}

Submit your manuscripts at

http://www.hindawi.com
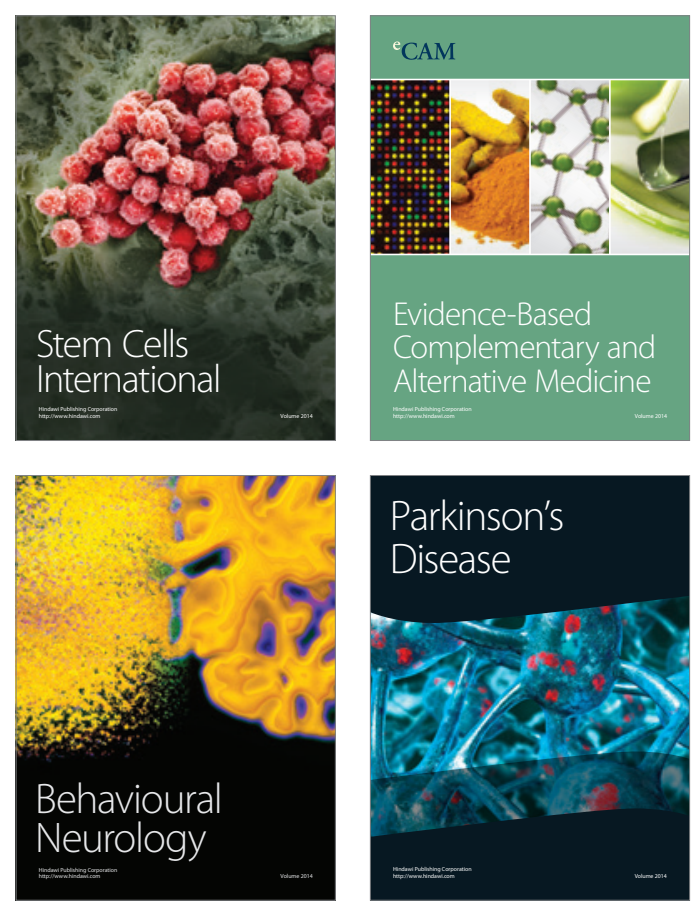

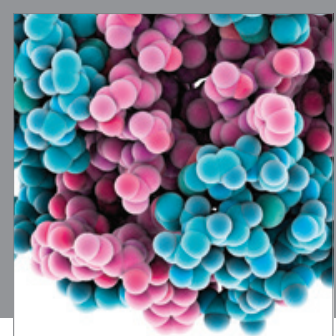

Journal of
Diabetes Research

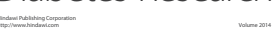

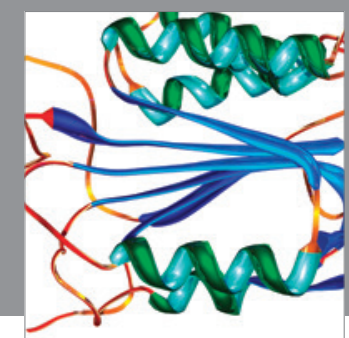

Disease Markers
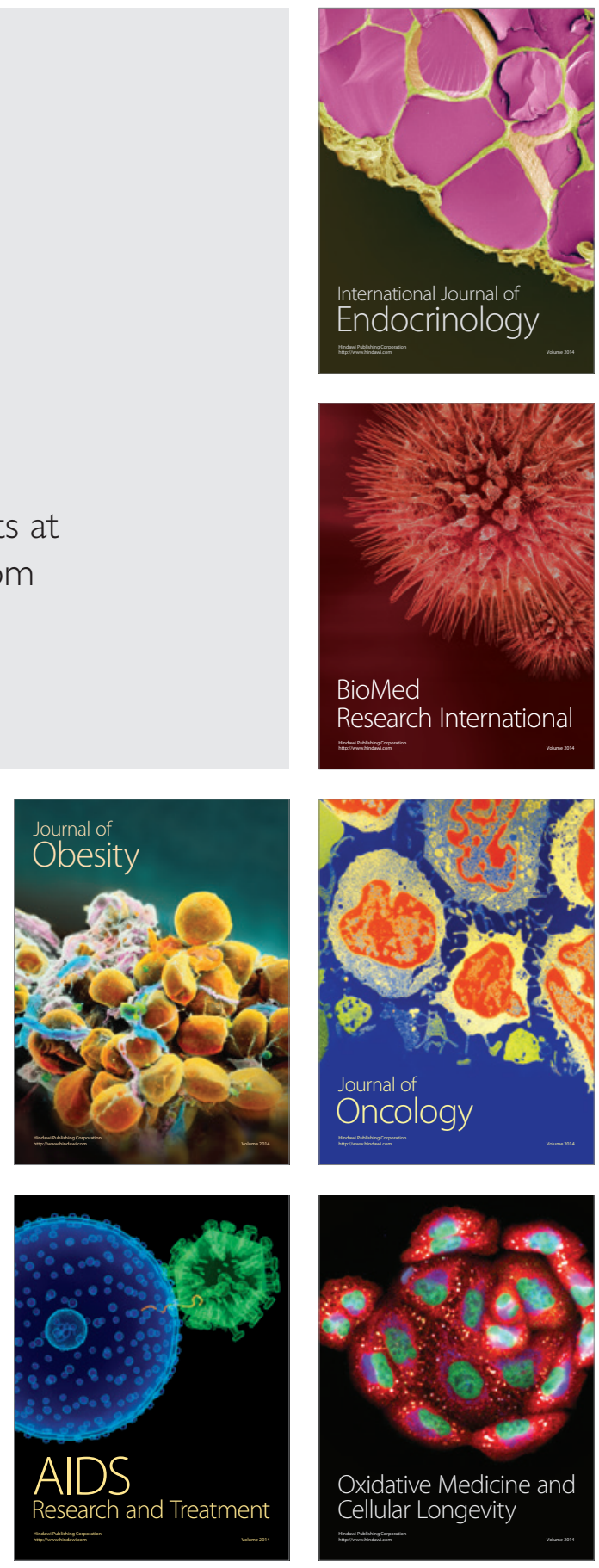